\title{
Resposta ao estresse: I. Homeostase e teoria da alostase
}

\author{
Maria Bernardete Cordeiro de Sousa \\ Hélderes Peregrino A. Silva \\ Nicole Leite Galvão-Coelho \\ Universidade Federal do Rio Grande do Norte
}

\section{Resumo}

Os seres vivos desenvolvem ao longo de sua história evolutiva mecanismos de enfrentamento às condições adversas originadas tanto no ambiente geofísico como no ambiente social. Esta resposta adaptativa é coordenada e envolve diferentes sistemas funcionais, particularmente, os sistemas nervoso, endócrino e imune, e é denominada de resposta ao estresse e deve atender a duas demandas principais da vida: sobrevivência e reprodução. Esta revisão tem o objetivo de discutir o emprego do conceito clássico de homeostase e um conceito alternativo, alostase, que inclui os mecanismos preditivos e reativos de regulação, assim como os diferentes níveis de impacto dos estressores crônicos, resultando em sobrecarga alostática que pode ou não se seguir de falha alostática. Os mecanismos neurais, hormonais, imunes, sistêmicos e moleculares, que compreendem os sistemas alóstaticos subjacentes à resposta ao estresse são também apresentados.

Palavras-chave: estresse; adaptação; eixo hipotálamo-pituitária-adrenal; cortisol; sistema neurovegetativo; sistema imune.

\begin{abstract}
Stress response: I. Homestasis and allostasis theory. Over the course of their evolution organisms developed mechanisms to cope with challenging situations originating from both physical and social environments. These adaptive mechanisms are coordinated and involve the sequential and/or simultaneous recruitment of different functional systems, mainly neural, endocrine and immune, the so-called stress response. They operate to maintain physiological variables at suitable. This review aims to discuss the use of the classical homeostasis concept and allostasis, an alternative model, which includes reactive and predictive mechanisms, as well as the different levels of impact of the chronic stressor, resulting in allostatic overload or allostatic failure. The organizing role of neural, hormonal, immune, systemic and molecular mechanisms, which comprise the allostatic systems underlying the stress response, is also presented.
\end{abstract}

Keywords: stress; adaptation; hypothalamus-pituitary-adrenal axis; cortisol; neurovegetative system; immune system.

\section{Resumen}

Respuesta al estrés: I. Homeostasis y teoría de la alostasis. Los seres vivos desarrollan a lo largo de su historia evolutiva mecanismos de enfrentamiento a las condiciones adversas originadas tanto en el ambiente geofísico como en el ambiente social. Esta respuesta adaptativa es coordinada y envuelve distintos sistemas funcionales, particularmente, el sistema nervioso, endócrino e inmune y es denominada de respuesta al estrés y debe atender a las dos demandas principales de la vida: supervivencia y reproducción. Esta revisión tiene como objetivo de discutir el empleo del concepto clásico de homeostasis y una concepto alternativa, alostasis, que incluye los mecanismos y el impacto de los agentes de estresores crónicos resultando en sobrecarga alostática o falla alostática. Los mecanismos neuronales, hormonales e inmunes, sistémicos y moleculares que comprenden los sistemas alóstaticos subyacentes a la respuesta al estrés son también presentados.

Palabras clave: estrés; adaptación; eje hipotálamo-hipófisis-suprarrenal; cortisol; sistema neurovegetativo; sistema inmunológico. 
$\mathrm{D}$ urante o processo evolutivo os seres vivos passaram por situações de desafios, que exigiam a ativação de respostas de enfrentamento, assim como a seleção de respostas adaptativas. As adaptações são processos que auxiliam os indivíduos a se adequarem ao ambiente, contribuindo para o aumento de sua sobrevivência e/ou reprodução.

O conceito de homeostase foi desenvolvido por Walter Cannon, pesquisador americano que estudava o aparelho digestório e o sistema nervoso autônomo. Cannon desenvolveu sua carreira científica na Universidade de Harvard e segundo seus biográfos possuia referências científicas nas figuras de Claude Bernard e Charles Darwin (Bennison et al., 1987). Realizou trabalhos em fisiologia experimental denominando de homeostase o princípio de "meio interno", descrito por C. Bernard (1911), de que "o sangue e os demais fluidos que circundam as células constituem o meio interno com o qual ocorrem as trocas diretas de cada célula e, por isto, deve ser mantido sempre com parâmetros adequados à função celular, independente das mudanças que possam estar ocorrendo no ambiente externo". Assim, propôs que a função final de todos os mecanismos fisiológicos é a manutenção da homeostase, que deve ser compreendida como "a manutenção da estabilidade do meio interno" (Cannon, 1929).

O fato de W. Cannon, antes mesmo de formalizar o conceito de homeostase, já se interessar pela investigação dos efeitos das emoções sobre a resposta fisiológica dos organismos, tendo introduzido a expressão "reação de luta ou fuga" (fight or flight response), cujo significado indica as reações autonômicas que se expressam numa situação desafiadora [Walter Bradford Cannon (1915). Bodily Changes in Pain, Hunger, Fear and Rage: An Account of Recent Researches into the Function of Emotional Excitement.]; e de que tanto o conceito de homeostase quanto o conceito de resposta de estresse tratam do entendimento dos mecanismos de regulação e ajuste do organismo frente a desafios, demonstra o quanto estes dois conceitos estão intimamente interligados.

As sucessivas propostas de revisão e ampliação do conceito de homeostase para contemplar tanto respostas antecipatórias e recorrentes quanto ajustes imediatos frente a situações extremas e imprevisíveis aproximam ainda mais os dois conceitos, revelando para ambos um mesmo substrato fisiológico de sistemas regulatórios adaptativos, especializados em gerir as respostas fisiológicas frente às instáveis condições dos ambientes físico e social. Esta visão mais ampla e integradora é consolidada no conceito de alostase, apresentado e discutido adiante. Para o melhor entendimento desta evolução conceitual, são apresentadas ao longo do texto, primeiramente, a emergência do conceito clássico de resposta de estresse, e subsequentemente, os ajustes e implicações do conceito de homeostase e a proposição da teoria da alostase.

\section{A resposta ao estresse e os sistemas alostáticos}

\section{Resposta ao estresse}

Conforme exposto anteriormente, o uso do conceito de estresse demanda uma compreensão dos mecanismos fisiológicos que geram uma resposta integrada de enfrentamento a situações adversas que remetemos ao conceito clássico de homeostase.
A ideia integradora desenvolvida por Walter Cannon de regulação das funções orgânicas a partir da manutenção do meio interno nos seres vivo teve influência nas conclusões de Hans Seyle, o primeiro pesquisador a utilizar o termo estresse em biologia. Seyle (1946) conceituou estresse como "a resposta não específica do corpo a qualquer demanda, seja ela causada por, ou resultando, em condições favoráveis ou não favoráveis”. A estas reações Selye denominou Síndrome da Adaptação Geral (SAG), com três fases distintas e identificadas atualmente como: (1) Alarme ou alerta - onde há a ruptura do equilíbrio interno do organismo e a mobilização do mesmo para enfrentar o agente estressor. Esta resposta rápida é mediada principalmente pela ativação do sistema nervoso autônomo simpático (SNAs) que promove a liberação de neurotransmissores em diversos órgãos-alvo e também estimula a medula das glândulas adrenais a liberarem os hormônios catecolaminérgicos, adrenalina e noradrenalina, reforçando ainda mais a ativação neural; (2) Resistência - as respostas fisiológicas e comportamentais, a fim de restabelecer a homeostase são mantidas, e agora mediadas principalmente pelo cortisol, hormônio esteroide sintetizado e liberado pelo córtex das glândulas adrenais, em resposta a ativação do eixo hipotálamo-pituitária-adrenal (HPA). Nesta fase a eficiência das respostas chega ao seu ponto máximo e o indivíduo apresenta seu melhor desempenho físico e cognitivo, e assim apresenta ampla condição de neutralizar o agente estressor; (3) Exaustão - caso o indivíduo falhe em neutralizar o agente estressor e este se prolongue, o organismo continua respondendo de forma crônica, e as alterações fisiológicas e comportamentais, inicialmente adaptativas, levam a uma sobrecarga energética e exaustão dos sistemas (Nelson, 2000). A conceituação da palavra estresse, portanto, é utilizada para indicar o conjunto de alterações fisiológicas, comportamentais, com repercussões no sistema imune, que o organismo desenvolve diante de um desafio (agente estressor) de natureza física ou psicossocial (positivo ou negativo), que rompe a homeostase do organismo e exige assim um esforço de adaptação (Zimpel, 2005).

As discussões recentes, entretanto, não encontram neste esquema que enfatiza unicamente a inespecificidade da resposta, a flexibilidade para explicar a resposta do homem e dos animais a desafios previsíveis como, por exemplo, uma generalização que compreenda as respostas fisiológicas ligadas aos fenômenos da reprodução e de respostas antecipatórias a eventos naturais. Além disso, sabe-se hoje que a resposta a estímulos estressores pode variar, em função do nível de previsibilidade, da intensidade, duração e natureza do estímulo estressor, e até mesmo em função das expectativas do sujeito (McEwen, 2002).

As revisões ao conceito de homeostase, discutindo e elucidando a complexidade e a diversidade dos mecanismos subjacentes à capacidade de adaptação dos organismos, vem contribuindo decisivamente para o entendimento da resposta de estresse. Entre os aspectos não contemplados no conceito clássico de homeostase, a discussão acerca das oscilações que ocorrem nos sistemas biológicos ocorreu nas décadas de 70 e 80 e foi revisada por Menna-Barreto (2004). Este autor se refere ao trabalho de Reinberg (1977) no qual o autor critica o conceito clássico de homeostase e propõe a própria negação deste conceito. Por outro lado, diante das evidências da existência da ritmicidade biológica e de mecanismos 
preditivos, Moore-Ede (1986) propõe a ampliação do uso do conceito de homeostase e dicotomiza o seu emprego em homeostase reativa (clássica) e homeostase preditiva. Esta última definição incluiria, especificamente, a temporização de mecanismos biológicos, de natureza circadiana e circanual que, de maneira preditiva, auxilia os organismos a responderem de maneira adequada às mudanças ambientais naturais como a alternância dos dias e das noites e das estações do ano. Estas respostas são imprescindíveis para a evolução de mecanismos adaptativos de regulação.

Outro autor que também considerou a importância das oscilações dos sistemas biológicos foi Mrozovsky (1990) quando debate a explicação de Walter Cannon no uso do termo homeostase (homeo= similar) e não homostase (homo= igual) e menciona que Cannon reconhece variação no controle dos sistemas biológicos e flexibiliza o conceito. Mrozovsky (1990) propõe o emprego do termo Reostase para indicar "a condição ou estado no qual, a qualquer instante, as defesas homeostáticas estão ainda presentes mas, sob um lapso de tempo, há uma modificação no nível de regulação da variável”. Deste modo, o termo Reostase (reos= mudança) indica a mudança no ponto de regulação da variável. Ou seja, o conceito de reostase propõe que o "valor de ajuste" de uma variável pode não ser constante, sofrendo mudanças associadas não só às variações cíclicas do ambiente (reostase programada - tendo como exemplo a hibernação, na qual a temperatura corporal é ativamente diminuída não ocorrendo como efeito da diminuição da temperatura do ambiente), ao desenvolvimento ontogenético (p. ex. puberdade), mas também associadas às variações não previsíveis (reostase reativa - como a febre que acompanha processos infecciosos) e, muito frequentemente, aos processos reprodutivos. Entre os exemplos citados pelo autor estão a variação de peso corporal que acompanha o ciclo estral em fêmeas de muitas espécies, a variação da temperatura corporal durante a fase luteal do ciclo menstrual e a diminuição da osmolaridade plasmática que acompanha a gravidez em mulheres. O conceito de reostase amplia o conceito de homeostase, enfatizando que adequações do "valor de ajuste" faz parte da forma de atuação dos mecanismos homeostáticos.

\section{O conceito de alostase}

A revisão do conceito de homeostase foi também proposta em 1988, com a utilização do termo alostase por Peter Sterling. Este autor aponta o encéfalo como órgão central regulador da alostase, tanto na regulação dos mecanismos periféricos, como naqueles que comandam os comportamentos nos níveis superiores e que aumentam a capacidade do organismo responder de maneira eficiente às suas necessidades. Diferentemente dos sistemas homeostáticos que priorizam a regulação por meio de mecanismos de retroalimentação negativa, os sistemas alostáticos dão relevância também aos mecanismos de regulação por retroalimentação positiva e por meio de ajustes antecipatórios que podem funcionar acoplados e simultaneamente em diferentes sistemas fisiológicos. Dessa forma, além de: (a) identificar e corrigir os erros, os sistemas alostáticos dispõem de meios para (b) produzirem um ajuste estreito e que serve a eficiência do mecanismo, prevendo, integrando e ajustando demandas, (c) dividir recursos entre os sistemas fisiológicos, evitando assim a exaustão de alguns sis- temas e subutilização de outros, e (d) armazenar informações de erros e minimizar tanto a magnitude quanto a frequência dos mesmos em momentos posteriores (Sterling, 2012).

Os sistemas de regulação homeostática visam restabelecer o equilíbrio do indivíduo (McEwen \& Wingfield, 2003; Troisi, 2001) durante as condições normais de regulação para manutenção do organismo em harmonia com situações que se alteram transitoriamente como, por exemplo, após a ingestão alimentar, durante o sono, durante a resposta sexual e reprodutiva. Todavia, quando ocorrem desafios impostos pelo ambiente social, físico, ou ambos, de maneira inesperada ou contínua, ultrapassando limites de intensidade, previsibilidade e duração, são ativados os sistemas regulatórios que passam a regular a homeostase em níveis mais elevados de demandas, o que McEwen (2000) a partir do conceito de Peter Sterling (Sterling \& Eyer, 1988) chamou de alostasia, e os respectivos mecanismos alostáticos.

Observa-se, portanto, que o conceito de alostase amplia e adequa o conceito de homeostase aos conhecimentos recentes da fisiologia, levando a uma nova visão também sobre a resposta ao estresse. Enquanto que o conceito clássico de homeostase prevê o funcionamento do organismo em condições basais, independentemente da demanda a qual o organismo está sujeito, a teoria da alostase distingue que os organismos podem funcionar em diferentes condições de ajustes, desde que estas novas condições possibilitem melhor adaptação dos organismos ao meio. A demanda para o estabelecimento de uma nova condição de ajuste pode ser desencadeada por agentes internos (mecanismos genéticos, neurais ou hormonais) ou externos (ambiente físico e social), previsíveis ou não, como acontece nos casos de incidência de um agente estressor (McEwen, 1998).

A ampliação do conceito de homestase a partir da "Teoria da alostase" de Sterling propõe que a ativação dos sistemas alostáticos gera um custo energético ao organismo, denominado de carga alostática que, quando em excesso, pode desencadear patologias físicas e transtornos mentais no indivíduo, situação esta conhecida como sobrecarga alostática (McEwen, 2003; McEwen \& Seeman 1999, 2000; McEwen \& Wingfield 2003a,b;). Recentemente, McEwen e Wingfield (2010) adicionaram um novo conceito a esta teoria: a falha alostática, que seria a incapacidade do indivíduo de ativar os sistemas alostáticos diante de uma demanda prolongada, que poderia desencadear processos patológicos, tendo em vista a inaptidão do organismo em responder adequadamente. A contribuição desta teoria está em demonstrar que a ativação dos sistemas alostáticos não ocorre apenas durante situações de crise, ou seja, de estresse, e que nem sempre desencadeia patologias. Tais sistemas podem e são ativados durante demandas previsíveis, vinculadas ao ciclo de vida do organismo, como também em situações de crise e inesperadas. Entretanto, se o custo energético desta ativação não for demasiado, não haverá prejuízo ao organismo, e nenhum processo patológico será desencadeado (McEwen, 2003; McEwen \& Wingfield, 2010).

McEwen (2000) e McEwen eWingfield (2003a) apresentam a importância do conceito de ativação alostática para a neuropsicofarmacologia e medicina, respectivamente, demonstrando como a teoria da alostase tem o potencial de integrar conhecimentos de diversos campos das ciências biomédicas a fim de elucidar os processos de saúde-doença vinculados à ativação dos sistemas alostáticos. 
Outra contribuição positiva ao emprego do conceito de alostase é discutida por Schulkin (2003), uma vez que os mecanismos de regulação homeostática são utilizados com base em sistemas de retroalimentação (feedbacks) negativos. $\mathrm{O}$ autor traz à discussão a importância dos mecanismos de retroalimentação positiva para a regulação alostática, principalmente no nível molecular de regulação. Afirma também que o emprego do termo alostase reflete uma alternativa mais flexível tanto do ponto de vista do estudo do comportamento quanto da fisiologia, chamando também a atenção para o papel central do encéfalo na regulação dos sistemas fisiológicos periféricos da resposta ao estresse (sistema nervoso autônomo e ações hormonais e humorais).

Outros autores questionaram a base conceitual da alostase, como por exemplo, Dallman (2003) que afirmou diante da teoria proposta por McEwen e Wingfield (2003a), que esta não altera conceitualmente a compreensão dos conceitos de "estressor" (estímulo que evoca a resposta ao estresse) e "resposta ao estresse" (as mudanças que ocorrem no encéfalo e no corpo com o estresse), nem a natureza da resposta fisiológica associada, que envolve a expressão comportamental, e as respostas autonômica, neuroendócrina e imune. Neste caso a autora propõe a manutenção do uso do conceito de homeostase e apresenta, em analogia ao modelo de alostasia proposto por McEwen e Wingfield (2003a), uma estratégia de gradação da resposta ao estresse em 3 perfis: (1) rápida, transiente e moderada, compatível com a definição de alostase; (2) resposta mais lenta e mantida, ou seja, prolongada, equivalente à carga alostática e (3) resposta ao estresse na presença de um estressor prolongado com ativação mantida, equivalente à sobrecarga alostática.

Outra crítica ao conceito de alostase e carga alostática foi também expressa por Romero et al. (2009) na proposição da teoria do Modelo do Escopo Reativo. Neste caso os autores propõem a manutenção do termo homeostase, abrangendo quatro situações e conceitos que conferem a flexibilidade necessária ao conceito clássico de homeostase, a partir da terminologia: Homeostase preditiva (HP) para contemplar as respostas fisiológicas diante das variações decorrentes da ritmicidade biológica (circadiana e sazonal); Homeostase reativa (HR) que alcança as respostas às mudanças imprevistas ou às ameaças relacionadas ao ambiente; Sobrecarga homeostática (SH) englobando as alterações que ocorrem nos níveis ou concentrações acima da faixa da HR e Falência homeostática (FH) que compreende as alterações que ocorrem nos níveis ou concentrações abaixo da faixa da HP. Estas duas últimas representam efeitos patológicos que não são compatíveis com a saúde de curto ou longo prazos. Neste caso, a expectativa de que os novos conceitos tenham a abrangência para atender às diferentes situações de vida no ambiente natural e de laboratório é atendida, como nos pressupostas da teoria da alostase.

Diante da diversidade de propostas de mudança conceitual atrelada à nova nomenclatura para expressar a flexibilidade do conceito de homeostase, passando a incluir as perturbações à condição basal, representada pela resposta ao estresse, optou-se por seguir os conceitos propostos por Sterling, McEwen, Wingfield e colaboradores. Esta aceitação ocorre pela compreensão de que a mesma se adequa melhor a uma visão mais ampla no estudo da natureza, variedade e adaptações dos mecanismos fisiológicos de regulação, onde se destaca a resposta ao estresse nos seus aspectos fisiológicos e fisiopatológicos. Esta teoria considera ainda que os fatores estressores podem ser provenientes do ambiente físico (oscilações circadianas, sazonais) e social (econômico e cultural) e estão inseridos nos contextos adaptativos de sobrevivência e reprodução. Portanto, esta proposição atende a generalização que o conceito deve possuir para abranger a variação na ocorrência de desafios aos seres humanos e demais animais, tanto em contextos ecológicos como experimentais.

\section{Sistemas de regulação alostática}

Quando um indivíduo encontra-se numa potencial ou real situação desafiadora, previsível ou não, o hipotálamo, região basal do diencéfalo, constituído por diferentes núcleos de controle visceral e hormonal, ativa imediatamente dois dos principais sistemas de regulação alostática: a divisão simpática do sistema nervoso autônomo (SNAs) e o eixo hipotálamo-pituitária-adrenal (HPA). Ambos desencadeiam alterações fisiológicas e comportamentais provendo meios para o indivíduo superar o desafio. Por sua vez, esses dois sistemas modulam um terceiro, e não menos importante sistema alostático: o sistema imunológico (SI) (McEwen \& Seeman 1999). A maioria das informações sobre os aspectos da organização neural e os mecanismos moleculares subjacentes à resposta ao estresse apresentados nesta revisão foram obtidas a partir de modelos experimentais e participam de uma resposta conservada em todos os vertebrados, incluindo a espécie humana.

Sistema nervoso autônomo simpático (SNAs). A divisão simpática do sistema nervoso autônomo é ativada imediatamente em uma situação de crise. O hipotálamo envia sinais neurais que aumentam a ativação simpática central e periférica. Perifericamente, a ativação do SNAs causa vasoconstrição, elevando a pressão sanguínea, dilatação dos brônquios e pupila, e inibição da digestão. Além disto, o SNAs induz a atividade da medula das glândulas adrenais, as quais liberam adrenalina e noradrenalina na corrente sanguínea. Estes hormônios induzem a quebra do glicogênio hepático e dos músculos esqueléticos, a liberação de glicose na corrente sanguínea e inibem a secreção de insulina pelo pâncreas, elevando assim a glicemia (Sapolsky, 2002). Além disto, dão continuidade ao incremento do funcionamento do sistema cardiovascular e respiratório, para que a glicose possa ser transportada e oxidada, respectivamente, de forma mais eficiente. Como consequência, tais hormônios estimulam o metabolismo nos músculos esqueléticos e no encéfalo (Nelson, 2000). No âmbito do sistema nervoso central, durante a resposta ao estresse é observado um estado de alerta geral tanto cognitivo quanto comportamental (Dünser \& Hasibeder 2009) em função da ativação do locus coeruleus principal núcleo noradrenérgico do encéfalo, localizado na região rostral da ponte e que envia axônios para diversas regiões do encéfalo, como a amígdala, hipotálamo, hipocampo e córtex pré-frontal. Esta última estrutura faz parte do neocórtex, indicando que, embora a maioria das estruturas que organizam a resposta ao estresse faça parte de áreas evolutivamente mais antigas do encéfalo, as estruturas corticais mais recentes, como o córtex pré-frontal, se integram a esta circuitaria, conferido o componente que permite a avaliação consciente dos contextos e as caraterísticas da resposta compatíveis com os mesmos. 
Eixo hipotálamo-pituitária-adrenal (HPA) e sua regulação. A inervação noradrenégica que chega ao hipotálamo é um dos ativadores do mecanismo inicial de funcionamento do eixo HPA, a partir da ativação das células parvocelulares dos núcleos paraventriculares (PVN- paraventricular nuclei) do hipotálamo (Young, 2001). Inicialmente, ocorre a liberação do fator liberador de corticotrofina (CRF-corticotropin releasing factor) na região do infundíbulo da hipófise (ou pituitária). Esse fator é secretado no sangue do sistema porta-hipofisário e se liga a dois tipos de receptores rCRF1 e rCRF2, na adenohipófise, induzindo a síntese e liberação do hormônio adreno-corticotrófico (ACTH- adrenocorticotropic hormone). Este último, por sua vez, é secretado na circulação sistêmica e induz a síntese e a liberação de glicocorticóides pelo córtex da adrenal. A finalização da resposta ao estímulo estressor é feita por meio do controle do eixo HPA por mecanismos de retroalimentação negativa (Joels \& Baram, 2009).

A partir dos estudos de Herman e Cullinan (1997) foram propostas duas vias principais de ativação do eixo HPA: (i) a chamada via sistêmica, ativada por sinais viscerais transmitidos por projeções catecolaminérgicas do tronco encefálico, ou seja, uma resposta induzida essencialmente por estressores de natureza física, e (ii) a via processiva, ativada por estímulos de natureza psicossocial, ou por sua antecipação. A via processiva inclui a modulação do eixo HPA, mais precisamente do PVN por meio de áreas límbicas, como a amígdala, o hipocampo e o córtex pré-frontal, através das conexões destas áreas com neurônios gabaérgicos do núcleo de leito da estria terminal (BnST- basal nuclei of stria terminalis) localizado na área pré-óptica do hipotálamo. As vias provenientes da amígdala causam ativação do eixo HPA, inibindo os neurônios gabaérgicos no BnST, enquanto a modulação hipocampal gera um tônus inibitório sobre o eixo HPA, estimulando os neurônios gabaérgicos BnST.

Recentemente Radley (2012) propôs um novo modelo para explicar a modulação bifuncional (inibitória e excitatória) exercida pelo córtex pré-frontal medial (mPFC- medial prefrontal cortex) pré-limbico, sobre o eixo HPA em situações de relevância emocional. Segundo este modelo, a porção dorsal do $\mathrm{mPFC}$ envia estímulos excitatórios aos neurônios gabaérgicos BnST, causando inibição do eixo HPA, enquanto a porção ventral do mPFC, infra-límbico, envia estímulos excitatórios para neurônios não gabaérgicos da BnST, causando ativação do eixo HPA. Apesar desta última via ainda não ter sido caracterizada funcionalmente, já é bem estabelecido que o estresse crônico leve a diminuição no número de sinapses e na densidade de espículas dendríticas em neurônios hipocampais e no $\mathrm{mPFC}$, em paralelo ao aumento desses índices no núcleo basolateral da amígdala. Estes dados, em conjunto, sugerem que o estresse crônico, de natureza emocional, leva a um desequilíbrio entre as vias inibitórias e excitatórias que modulam a atividade do eixo HPA (Radley, 2012).

A partir da síntese e liberação dos glicocorticoides pelo córtex da adrenal, dos quais o cortisol é o principal representante nos primatas (Nelson 2000), a resposta ao estímulo estressor ativa dois tipos de receptores intracelulares: (1) receptor mineralocorticoide (MR- mineralocorticoid receptor) localizados no núcleo das células alvo com alta afinidade pelo cortisol e que são imprescindíveis para a expressão das respostas circadianas do cortisol (Joels \& Baram 2009); (ii) re- ceptor glicocorticoide (GR- glucocorticoid receptor) localizados no citoplasma e, quando ligados ao cortisol, migram sob a forma de dímeros para o núcleo. Estes últimos possuem menor afinidade pelo cortisol, porém participam ativamente do sistema de retroalimentação negativa do eixo HPA e respondem pelas alterações fisiológicas desencadeadas pelos níveis elevados de cortisol durante a resposta ao estresse (Anacker et al., 2013). Ambos os tipos de complexos cortisol-receptores interagem com sequências conhecidas como elementos responsivos aos glicocorticóides (GRE- glucocorticoid responsive element) em regiões promotoras de genes regulados por glicocorticóides, ativando ou reprimindo a transcrição de RNA mensageiro e a síntese proteica, o que resulta em lentas respostas sistêmicas, que incluem: aumento da glicemia (pelo incremento da gliconeogênese hepática); direcionamento deste aporte energético para as funções essenciais à situação de desafio, como as sensoriais, cognitivas e motoras; incremento no funcionamento dos sistemas cardiovascular e respiratório. Por outro lado, a digestão, o crescimento, a reprodução e a percepção à dor são inibidas (Falkenstein, Tillmann, Christ, \& Wehling, 2000). O cortisol também pode se ligar aos receptores de membrana, modulando a abertura e fechamento de canais iônicos e, dessa forma, desencadeando respostas fisiológicas rápidas como, por exemplo, alteração da liberação de neurotransmissores na fenda sináptica e, consequentemente, alteração da excitabilidade neuronal (Mora, Segovia, Del Arco, De Blas, \& Garrido, 2012; Riedemann et al., 2010).

A finalização da resposta ao estímulo estressor (recovery) é realizada por meio do controle do eixo HPA por mecanismos de retroalimentação negativa. Herman e colaboradores (2012), em uma revisão sobre os mecanismos de retroalimentação negativa do eixo pelos glicocorticóides, identificaram mecanismos lentos, genômicos, já bem conhecidos e mecanismos rápidos, não-genômicos. A ligação do cortisol aos seus receptores intracelulares clássicos, principalmente ao GR, na hipófise, hipotálamo, hipocampo e no córtex pré-frontal medial dorsal inibem a ativação do eixo HPA (Ulrich-Lai \& Herman, 2009; Zigmond et al., 1999) por modificações na transcrição gênica sendo, portanto, mecanismos de ação lenta (Herman et al., 2012). Adicionalmente, as regiões límbicas acima citadas apresentam uma grande população de receptores para o $\mathrm{CRF}$, que nestas regiões atuam coordenando a atividade das vias que modulam as respostas neuroendócrina, autonômica e comportamental ao estresse, de forma independente de sua atuação na estimulação direta sobre o PVN. Em um estudo em ratos no qual apenas os circuitos límbicos do CRF foram inativados, Muller e colaboradores (2003) observaram que a ligação do $C R F$ aos receptores $r C R F-1$ na amígdala produz efeito ansiolítico e contribui para a retroalimentação negativa do eixo HPA, através do controle sobre a expressão de MR no hipocampo, importante no processo de adaptação da resposta ao estresse. Um estudo em ratos nocauteados para as urocortinas (neuropeptídeos da família do CRF e ligantes preferenciais do receptor CRF-2) sugere que a ativação dos receptores CRF-2 permite a diminuição gradativa dos níveis de ansiedade que tenham sido elevados por exposição a um estímulo estressor, pois estes animais geneticamente modificados apresentam incapacidade de recuperar os níveis basais de ansiedade 24 horas após o evento estressor. Além disso, ocorre um comprometimento da regulação gênica de circuitos inibitórios na 
amígdala, envolvidos na resposta de estresse (Neufeld-Cohen et al., 2010). A apresentação desses dois tipos de receptores na amígdala apresenta-se sexualmente dimórfica a partir da puberdade, o que tem sido relacionado com as diferenças entre os gêneros na susceptibilidade a desenvolver desordens de humor e ansiedade (Weathington \& Cooke, 2012).

O mecanismo de retroalimentação negativa rápida sobre o eixo HPA ocorre quando o cortisol liga-se a seus receptores de membrana nas células parvocelulares do PVN hipotalâmico, com a participação dos endocanabinóides, que são neurotransmissores retrógrados liberados pelos neurônios pós-sinápticos, em resposta a presença do cortisol. Os endocanabinóides atuam através da ligação aos receptores CB1 pré-sinápticos, inibindo a abertura de canais de cálcio voltagem dependentes, bloqueando o influxo deste íon e a liberação de glutamato pelos respectivos neurônios pré-sinápticos, reduzindo assim a atividade no PVN e, consequentemente, na ativação do eixo HPA (Herman et al., 2012; Saito et al., 2010). Sendo assim, os endocanabinóides possuem efeitos ansiolíticos, uma vez que exercem um tônus inibitório sobre o funcionamento do eixo HPA (Cota, 2008).

Hill e Tasker (2012) sugerem que o endocanabinóide anandamida, em condições basais, exerce um tônus inibitório sobre a ativação da amígdala, através da sua ligação a receptores CB1 e redução na liberação glutamatérgica, reduzindo assim a ativação do eixo HPA. Em situações de estresse agudo, possivelmente através do CRF ou noradrenalina, os mecanismos de hidrólise da anandamida são incrementados ocorrendo maior liberação glutamatérgica, ativação da amígdala e consequentemente do eixo HPA. A liberação do cortisol resultante da ativação do eixo HPA estimula novamente a síntese e liberação da anadamida que por sua vez volta a reduzir a ativação do eixo HPA (Hill et al., 2010). Patel et al. (2004) observaram, numa situação de estresse físico agudo, em roedores, a ativação do eixo HPA e a redução do endocanabinóide glicerol 2-araquidonoil (2-AG) e posteriormente, durante a fase de adaptação ao agente estressor, a atenuação dos níveis de cortisol, concomitantemente ao aumento dos níveis deste endocanabinóide. Herman et al. (2012) sugerem que mecanismos de desestabilização e degradação dos RNA mensageiros também podem participar do controle rápido da retroalimentação negativa do eixo HPA.

Outros importantes mensageiros químicos cerebrais também são ativados durante a reposta ao estresse e atualmente são vistos como importantes moduladores desta resposta, como por exemplo; vasopressina, ocitocina, dopamina, serotonina e óxido nítrico, além do fator neurotrófico derivado do encéfalo (BDNF- brain derived neurotrophic fator) (Joels \& Baram, 2009).

Em resumo, a resposta ao estresse apresenta níveis de organização decorrentes dos mecanismos rápidos da transmissão sináptica aminérgica e peptidérgica e liberação hormonal, correspondente às ações da adrenalina, noradrenalina e liberação do CRF que produz os comportamentos de alerta, vigilância e as escolhas de uma ou mais estratégias adequadas para fazer frente ao desafio (Joels \& Baram, 2009). Estas ações rápidas são seguidas pela ativação de genes de expressão imediata e envolvem também a participação do cortisol em vias não genômicas no hipotálamo e no hipocampo. Nesta última estrutura o cortisol atua em receptores $\mathrm{MR}$, como demonstrado em modelos experimentais. Posteriormente ocorrem ações genômicas e efeitos estruturais responsáveis pela resposta mais longa.

Sistema imunológico (SI). Estudos apontam a existência de conexões bidirecionais entre o SNAs, o eixo HPA e o sistema imunológico (Marques, Silverman, \& Sternberg 2010; Salposky, 2002). O SI modula os sistemas alostáticos através das citocinas, que são proteínas sinalizadoras de ação pleiotrópica (Montoro et al., 2009). Algumas citocinas exercem efeitos estimulatórios sobre o SNA e eixo HPA, enquanto outras exercem efeitos inibitórios. Sabe-se que a interleucina 1 (IL-1) induz a liberação do fator liberador de corticotrofina (CRF) no hipotálamo (Segerstrom, 2010; Uchino, Kiecolt-Glaser, \& Glaser, 2000) e também parece ser importante para o desenvolvimento da resistência dos receptores de glicocorticóides ao cortisol durante o estresse crônico (Zunszain, Anacker, Cattaneo, Carvalho, \& Pariante 2011).

A medula óssea e grande parte dos órgãos linfóides são inervadas pelo SNAs e as células do sistema imunológico apresentam receptores para diversos neurotransmissores e hormônios envolvidos na resposta ao estresse, possibilitando uma neuromodulação direta sobre os aspectos celulares da resposta imune (Uchino et al., 2000). Mais especificamente, a liberação de adrenalina e noradrenalina durante a fase de alerta estimulam uma rápida produção de neutrófilos e células exterminadora natural (NK- natural killers) do sistema imune inato, permitindo que o indivíduo elimine de forma mais eficiente eventuais infecções provenientes da resposta ao estresse (Segerstrom, 2010). Essas catecolaminas também ativam a proliferação de linfócitos T citotóxicos (TCD8), células do sistema imune adquirido (Uchino et al., 2000).

Os hormônios glicocorticóides por sua vez influenciam o funcionamento do SI de acordo com sua concentração e duração na sua atuação. Níveis moderados de cortisol, liberados durante o estresse agudo, induzem a redistribuição dos linfócitos, desviando-os do sangue para os tecidos mais susceptíveis a infecções (Sapolsky, 2002). Induz ainda o deslocamento da produção de linfócitos T auxiliar (TCD4) Th1 para a Th2, propiciando um ambiente rico em citocinas anti-inflamatórias, linfócitos B e eosinófilos, e suprimindo a produção de citocinas inflamatórias e células TCD8 (Montoro et al., 2009). Esta ação anti-inflamatória auxilia no retorno do funcionamento do sistema imune aos seus níveis basais, previamente incrementados pela ação das catecolaminas (Baumann \& Turpin, 2010). Entretanto, a exposição prolongada ao cortisol pode provocar um desequilíbrio nas funções imunológicas resultando em imunodeficiência. Inicialmente o hipercortisolismo induz uma hiperativação da resposta Th2, e este quadro imunológico pode resultar em maior suscetibilidade do organismo ao desenvolvimento de alergias e infecções virais. Num segundo momento a ativação crônica do sistema imunológico Th2, do eixo HPA e o hipercortisolismo podem alterar a sensibilidade dos receptores de glicocorticóides das células do sistema imune. Nesta situação há um deslocamento da resposta Th2 para a Th1, ocorrendo uma redução na proliferação de linfócitos B e das citocinas anti-inflamatórias, interleucina 4 (IL-4) e interleucina 10 (IL-10), e uma elevação na concentração de citocinas pro-inflamatórias IL-1 e interleucina 6 (IL-6) e do fator de necrose tumoral alfa (TNF- $\bigotimes$ ) (Marques, Cizza, \& Sternberg 2007; Sanders et 
al.,2000; Zunszain et al., 2011). Este quadro de desequilíbrio e a formação de um ambiente rico em citocinas pro-inflamatórias é frequentemente relacionado à etiologia de algumas patologias cerebrais, como a depressão e o Mal de Alzheimer (Kapadia \& Sakic, 2011; Maes et al., 2009).

Evidências recentes em roedores demonstram que o recrutamento de monócitos no encéfalo durante o estresse social constitui também um mecanismo celular que pode contribuir para o desenvolvimento de ansiedade (Wohleb et al., 2013).

Em resumo, a ativação dos sistemas de resposta ao estresse diante um agente estressor consiste em um fenômeno comum a todos os vertebrados, com características fisiológicas semelhantes, decorrente de variações adaptativas que envolvem a integração de influências genéticas (estrutura geral dos sistemas) e uma larga variabilidade na reatividade destes sistemas entre os indivíduos (diferenças individuais) (Ellis, Jackson, \& Boyce, 2006). Compreende, portanto, uma resposta coordenada dos mediadores do estresse que envolve a participação do sistema nervoso autônomo, sistema endócrino e de natureza imunológica. Todavia, as respostas fisiológicas e comportamentais disparadas por estes sistemas não são iguais entre as espécies, nem mesmo entre os indivíduos de uma mesma espécie (Orchinik, 1998), ou no mesmo indivíduo em diferentes situações.

O impacto causado por um agente estressor, ou seja, a intensidade e a duração da ativação dos sistemas alostáticos de um organismo são determinadas em parte por características do próprio estímulo (natureza, tempo de exposição, previsibilidade), e em parte pela habilidade que o organismo possui em lidar com a situação (Korte, Koolhaas, Wingfield, \& McEwen, 2005; McEwen 1998), ou seja, da plasticidade fenotípica. Essa resposta está diretamente correlacionada a fatores individuais (sexo, idade, temperamento, controlabilidade sobre o agente estressor (McEwen, 2002; Peters et al., 1998; Tyrka et al., 2008) e sociais (posto e/ou suporte sociais) (McEwen, 2007). Decorrente deste fato, algumas pessoas apresentam a capacidade de enfrentamento diante de dificuldades, conhecido como resiliência (Charmandari et al., 2005; Sapolsky, 2000) enquanto outros indivíduos são mais vulneráveis a desenvolver patologias físicas ou mentais decorrentes da resposta crônica aos agentes estressores.

O conceito de resiliência e suas implicações serão debatidos na parte II desta revisão. Esta discussão é fundamental para a compreensão dos mecanismos de enfrentamento das situações de estresse pelos organismos e as suas consequências que podem ser atenuadas ou não, neste último caso, desencadeando diferentes patologias.

\section{Referências}

Anacker, C., Cattaneom, A., Luoni, A., Musaelyan, K., Zunszain, P. A., Milanesi, E., ... Pariante, C. M. (2013). Glucocorticoid-related molecular signaling pathways regulating hippocampal neurogenesis. Neuropsychopharmacology; 38, 872-883. doi:10.1038/npp.2012.253

Baumann, N., \& Turpin, J. C. (2010). Neurochemistry of stress: An overview. Neurochemistry Research, 35(12), 1875-1879. doi: 10.1007/s11064-010-0298-9

Bennison, S., Barger, A. C., \& Wolfe, E. L. (1987). Walter B. Cannon: The life and times of a young scientist. Cambridge, MA: Harvard University Press.
Bernard, C. (1911). La science expérimentale. Paris: Libr. J-B Baillière et Fils.

Cannon, W. B. (1915). Bodily Changes in Pain, Hunger, Fear and Rage: An Account of Recent Researches into the Function of Emotional Excitement. Nova Iorque: D. New Appleton and Company.

Cannon, W. B. (1929). Organization for physiological homeostasis. Physiolog ical Reviews, 9(3), 399-403. Recuperado de http://physrev.physiology. org/content/physrev/9/3/399.full.pdf

Charmandari, E., Tsigos, C., \& Chrousos, G. (2005). Endocrinology of the stress response. Annual Review of Physiology, 67, 259-284. doi: 10.1146/ annurev.physiol.67.040403.120816

Cota, D. (2008). The role of the endocannabinoid system in the regulation of hypothalamic-pituitary-adrenal axis activity. Journal of Neuroendocrinology, 1, 35-38. doi: 10.1111/j.1365-2826.2008.01673.x

Dallman, M. F. (2003). Stress by any other name? Hormones and Behavior, 43(1), 18-20. doi:10.1016/S0018-506X(02)00034-X

Dünser, M. W., \& Hasibeder, W. R. (2009). Sympathetic overstimulation during critical illness: Adverse effects of adrenergic stress. Journal of Intensive Care Med, 24(5), 293-316. doi: 10.1177/0885066609340519

Ellis, B. J., Jackson, J. J., \& Boyce, W. T. (2006). The stress response systems: Universality and adaptive individual differences. Developmental Review, 26(2), 175-212. doi:10.1016/j.dr.2006.02.004

Falkenstein, E., Tillmann, H. C., Christ, M., \& Wehling, M. (2000). Multiple actions of steroid hormones - a focus on rapid, nongenomic effects. Pharmacological Reviews, 52(4), 513-555. doi: 0031-6997/00/52040513\$03.00/0

Herman, J. P., \& Cullinan, W. (1997). Neurocircuitry of stress: Central control of the hypothalamo-pituitary-adrenocortical axis. Trends in Neuroscience, 20(2), 78-84. doi:10.1016/S0166-2236(96)10069-2

Herman, J. P., McKlveen, J. M, Solomon, M. B., Carvalho-Neto, E., \& Myers, B. (2012). Neural regulation of the stress response: Glucocorticoid feedback mechanisms. Brazilian Journal of Medical and Biological Research, 45(4), 292-298. doi:10.1590/S0100-879X2012007500041

Hill, M. N., \& Tasker, J. G. (2012). Endocannabinoid signaling, glucocorticoid-mediated negative feedback and regulation of the HPA axis. Neuroscience, 204, 5-16. doi: 10.1016/j.neuroscience.2011.12.030

Hill , M. N., McLaughlin, R. J., Bingham, B., Shrestha, L., Lee, T. T, Gray, J. M., ... Viau, V. (2010). Endogenous cannabinoid signaling is essential for stress adaptation. Proceedings of the National Academy of Sciences of the United States of America, 107, 9406-11. doi: 10.1073/ pnas.0914661107

Joels, M., \& Baram, T. Z. (2009). The neuro-symphony of stress. Nature, 10(6), 459-466. doi: 10.1038/nrn2632

Kapadia, M., \& Sakic, B. (2011). Autoimmune and inflammatory mechanisms of CNS damage. Progress in Neurobiology, 95(3), 301-33. doi: 10.1016/j. pneurobio.2011.08.008

Korte, S. M., Koolhaas, J. M., Wingfield, J. C., \& McEwen, B. S. (2005). The Darwinian concept of stress: Benefits of allostasis and costs of allostatic load and the trade-offs in health and disease. Neuroscience and Biobehavioral Reviews, 29(1), 3-38. doi:10.1016/j.neubiorev.2004.08.009

Maes, M., Yirmyia, R., Noraberg, J., Brene, S., Hibbeln, J., Perini, G., ... Maj, M. (2009). The inflammatory \& neurodegenerative (I\&ND) hypothesis of depression: Leads for future research and new drug developments in depression. Metabolic Brain Disease, 24(1), 27-53. doi: 10.1007/s11011008-9118-1

Marques, A. H, Cizza, G., \& Sternberg, E. (2007). Brain-immune interactions and implications in psychiatric disorders. Revista Brasileira de Psiquiatria, 1, S27-32. doi: 10.1590/S1516-44462007000500006

Marques, A. H., Silverman, M. N., \& Sternberg, E. M. (2010). Evaluation of stress systems by applying noninvasive methodologies: Measurements of neuroimmune biomarkers in the sweat, heart rate variability and salivary cortisol. Neuroimmunomodulation, 17(3), 205-208. doi $10.1159 / 000258725$ 
McEwen, B. S. (1998). Stress, adaptation and disease: Allostasis and allostatic load. Annals of the New York Academy of Science, 840, 33-44. doi: 10.1111/j.1749-6632.1998.tb09546.x

McEwen, B. S. (2000). Allostasis and allostatic load: Implications for neuropsychopharmacology. Neuropsychopharmacology, 22(2), 108-124. doi:10.1016/S0893-133X(99)00129-3

McEwen, B. S. (2002). Sex, stress and the hippocampus: Allostasis, allostatic load and the aging process. Neurobiology of Aging, 23(5), 921-39. doi:10.1016/S0197-4580(02)00027-1

McEwen, B. S. (2003). Mood disorders and allostatic load. Biological Psychiatry, 54, 200-207. doi:10.1016/S0006-3223(03)00177-X

McEwen, B. S. (2007). Physiology and neurobiology of stress and adaptation: Central role of the brain. Physiological Reiewsv, 87(3), 873-904. doi: 10.1152/physrev.00041.2006

McEwen, B. S., \& Seeman, T. (1999). Protective and damaging effects of mediators of stress elaborating and testing the concepts of allostasis and allostatic load. Annals of the New York Academy of Science, 869, 30-47. doi: 10.1111/j.1749-6632.1999.tb08103.x

McEwen, B. S., \& Wingfield, J. C. (2003a). The concept of allostasis in biology and biomedicine. Hormones and Behavior, 4(1), 2-15. doi:10.1016/ S0018-506X(02)00024-7

McEwen, B. S., \& Wingfield, J. C. (2003b). Response to commentaries on the concept of allostasis. Hormones and Behavior, 43(1), 28-30. doi:10.1016/ S0018-506X(02)00039-9

McEwen, B. S, \& Wingfield, J. C. (2010). What is in a name? Integrating homeostasis, allostasis and stress. Hormones and Behavior, 57(2), 105-11. doi:10.1016/j.yhbeh.2009.09.011

Menna-Barreto, L. (2004). Homeostasia: uma revisão necessária? Revista de Neurociências, 1(2), 105-107.

Montoro, J., Mullol , J., Jáuregui, I., Dávila, I., Ferrer, M., Bartra, J., ... Valero, A. (2009). Stress and allergy. Journal of Investigative Allergology and Clinical Immunollogy, 19(Suppl 1), 40-47. Recuperado de http://www.jiaci. org/issues/vol19s1/7.pdf

Moore-Ede, M. C. (1986). Physiology of the circadian timing system: Predictive versus reactive homeostasis. American Journal of Physiology, 250, R737-R752.

Mora. F., Segovia, G., Del Arco, A., De Blas, M., \& Garrido, P. (2012). Stress, neurotransmitters, corticosterone and body-brain integration. Brain Research, 1476, 71-85. doi: 10.1016/j.brainres.2011.12.049.

Mrozovsky, N. (1990). "Rheostasis -Tthe physiology of change". Nova Iorque, NY: Oxford University Press.

Muller, M. B., Zimmermann, S., Sillaber, I., Hagemeyer, T. P., Deussing, J. M., Timpl, P., ... Wurst, W. (2003). Limbic corticotropin-releasing hormone receptor 1 mediates anxiety-related behavior and hormonal adaptation to stress. Nature Neuroscience 6(10), 1100-1107. doi:10.1038/nn1123

Nelson, R. (2000). An introduction to behavioral endocrinology ( $2^{\mathrm{a}}$. ed.). Sunderland, MA: Sinauer Associates

Neufeld-Cohen, A., Tsoory, M. M., Evans, A. K., Getselter, D., Gil, S., Lowry, C. A., ... Chen, A. A. (2010). Triple urocortin knockout mouse model reveals an essential role for urocortins in stress recovery. Proceedings of the National Academy of Science of the United States of America, 107(44), 19029-19025. doi: 10.1073/pnas.1013761107

Orchinik, M. (1998). Glucocorticoids, stress, and behavior: shifting the timeframe. Hormones and Behavior, 34(3), 320-327. doi:10.1006/ hbeh.1998.1488

Patel, S., Roelke, C. T., Rademacher, D. J., Cullinan, W. E., \& Hillard, C. J. (2004). Endocannabinoid signaling negatively modulates stress-induced activation of the hypothalamic-pituitary-adrenal axis. Endocrinology, 145(12), 5431-8. doi: 10.1210/en.2004-0638
Peters, M., Godaert, G. L. R.., Ballieux, R. E., Vilet, M., Willemsen, J. J., Sweep, F. C. G. J., \& Heijnen, C. J. (1998). Cardiovascular and endocrine responses to experimental stress: Effects of mental effort and controllability. Psychoneuroendocrinoogy, 23(1), 1-17. doi:10.1016/S03064530(97)00082-6

Radley, J. J. (2012). Toward a limbic cortical inhibitory network: Implications for hypothalamic-pituitary-adrenal responses following chronic stress. Frontiers in Behavioral Neuroscience, 6, 1-10. doi: 10.3389/fnbeh.2012.00007

Reinberg, A. (1977). Des rythmes biologiques à la Chronobiologie. Paris: Bordas.

Riedemann, T., Patchv, A. V., Cho, K., \& Almeida, O. F. X. (2010). Corticosteroids: way upstream. Molecular Brain, 3(2), 1-20. doi:10.1186/1756$6606-3-2$

Romero, M. L., Dickens, M. J., \& Cyr, N. E. (2009). The reactive scope model - A new integrating homeostasis, allostasis and stress. Hormones and Behavior, 55(3), 375-389. doi:10.1016/j.yhbeh.2008.12.009

Saito, V. M., Wotjak, C. T., \& Moreira, F. A. (2010). Pharmacological exploitation of the endocannabinoid system: New perspectives for the treatment of depression and anxiety disorders? Revista Brasileira de Psiquiatria, 32(Suppl. 1), 57-514. doi: 10.1590/S1516-44462010000500004

Sanders, V. M., Iciek, L., \& Kasprowicz, D. J. (2000). Psychosocial factors and humoral immunity. In J. P Cacioppo, L. G. Tassinary, \& G. G. Berntson (Orgs.), Handbook of Psychophysiology (2a . ed., pp. 425-455). Nova Iorque, NY: Cambridge University Press.

Sapolsky, R. M. (2002). Endocrinology of the stress-response. In J. B. Becker Sl M. Breeedlove, D. Crews \& M. M McCarthy (orgs.), Behavioural Endocrinology (pp. 409-450). Cambridge, MA: MIT Press.

Sapolsky, R. M. (2000). Stress hormones: Good and bad. Neurobiology of Disease, 7(5), 540-2 doi: 10.1006/nbdi.2000.0350

Schulkin J. (2003). Allostasis: A neural behavioral perspective. Hormones and Behavior, 43(1), 21-27. doi:10.1016/S0018-506X(02)00035-1

Segerstrom, S. C. (2010). Resources, stress, and immunity: An ecological perspective on human psychoneuroimmunology. Annals of Behavioral Medicine, 40(1), 114-125. doi: 10.1007/s12160-010-9195-3

Selye H. (1946). The general adaptation syndrome and the diseases of adaptation. Journal of Clinical Endocrinology \& Metabolism, 6(2), 117-230. doi: 10.1210/jcem-6-2-117

Sterling, P. (2012). Allostasis: a model of predictive regulation. Physiology \& Behavior, 106, 5-15. doi: 10.1016/j.physbeh.2011.06.004

Sterling P., \& Eyer, J. (1988). Allostasis: A new paradigm to explain arousal pathology. In S. Fisher \& J. Reason (Orgs.), Handbook of life stress, cognition and health (pp. 629-649). Nova Iorque, NY: John Wiley \& Sons

Troisi, A. (2001). Gender differences in vulnerability to social stress: A Darwinian perspective. Physiology \& Behavior, 73(3), 443-449. doi:10.1016/ S0031-9384(01)00459-0

Tyrka, A., Mello, A. F., Mello, M. F., Gagne, G. G., Grover, K. E., Anderson, .. Carpenter, L. L. (2006). Temperament and hypothalamic-pituitary-adrenal axis function in healthy adults. Psychoneuroendocrinology, 31(9), 1036-1045. doi:10.1016/j.psyneuen.2006.06.004

Uchino, B. M, Kiecolt-Glaser, J. K., \& Glaser, R. (2000). Psychosocial factors and cellular immunity. In. O. J. P. Caciopp, L. G. Tassinary, \& G. G. Berntson (Orgs.), Handbook of Psychophysiology (397-424). Nova Iorque, NY: Cambridge University Press.

Ulrich-Lai, Y. M, \& Herman, J. P. (2009). Neural regulation of endocrine and autonomic stress responses. Nature Reviews Neuroscience, 10, 397-409. doi:10.1038/nrn2647

Weathington, J. M, \& Cooke, B. M. (2012). Corticotropin-realising factor receptor binding in the amygdala changes across puberty in a sex-specific manner. Endocrinology, 153(12), 5701-5705. doi: 10.1210/en.2012-1815

Wohleb, E. S., Powell N. D., Godbout, J. P., \& Sheridan, J. F. (2013). Stress-in- 
10 Resposta ao estresse: I. Homeostase e teoria da alostase

duced recruitment of bone marrow-derived monocytes to the brain promotes anxiety-like behavior. The Journal of Neuroscience, 33(34), 13820-13833; doi: 10.1523/JNEUROSCI.1671-13.2013

Young, L. T. (2001). Postreceptor pathways for signal transduction in depression and bipolar disorder. Journal of Psychiatry \& Neuroscience, 26 (Suppl. 17-22). PubMed: 11590965

Zigmond, M., Bloom, F. E., Landis, S. C., Roberts, J., \& Squire, L. (1999). Fundamental Neuroscience ( $1^{\mathrm{a}}$ ed.). Amsterdan: Academic Press.

Zimpel, R. R. (2005). Aprendendo a lidar com o estresse. São Leopoldo: Sinodal.

Zunszain, P. A., Anacker, C., Cattaneo, A., Carvalho, L. A., \& Pariante, C. M. (2011). Glucocorticoids, cytokines and brain abnormalities in depression. Progress in Neuro-Psychopharmacology and Biological Psychiatry, 35(3), 722-729. doi: 10.1016/j.pnpbp.2010.04.011 
Maria Bernardete Cordeiro de Sousa, Doutorado na Faculdade de Medicina- USP-Ribeirão Preto-SP, Pós-doutorado em Fisiologia Etológica na University of Wisconsin-USA, é professora Titular do Instituto do Cérebro da Universidade Federal do Rio Grande do Norte (UFRN). Endereço para correspondência: Universidade Federal do Rio Grande do Norte, Av. Nascimento de Castro, 2155 - 59056-450 , Natal/RN - Brasil. Telefone/fax: +55 (84) 32154592. E-mail: mbcsousa@neuro.ufrn.br

Hélderes Peregrino A. Silva, Doutorado em Psicologia Experimental pelo Instituto de Psicologia da Universidade de São Paulo - USP, é professor Associado I do Departamento de Fisologia na Universidade Federal do Rio Grande do Norte (UFRN). E-mail: helderes@cb.ufrn.br

Nicole Leite Galvão-Coelho, Doutora em Psicobiologia pela Universidade Federal do Rio Grande do Norte (UFRN), é professora Adjunta III do Departamento de Fisiologia da Universidade Federal do Rio Grande do Norte (UFRN). E-mail: nicolelgalvaocoelho@gmail.com 